Bio-grafía Escritos sobre la Biología y su Enseñanza.

Edición Extra-Ordinaria.

Memorias del I Congreso Nacional de Investigación en Enseñanza de la Biología.

VI Encuentro Nacional de Investigación en Enseñanza de la Biologia y la Educación Ambiental. ISSN 2027 1034. P. p. 320-332.

\title{
Acercamiento a la historia de la instalación de la biotecnología en la educación
}

\section{Approaching to the history of biotechnology installation education}

POR: Robinson Roa Acosta ${ }^{1}$

\section{Resumen}

En este escrito se encuentran aspectos relacionados con la llegada de la biotecnología a la educación. Es resultado de la búsqueda y revisión minuciosa a los primeros documentos publicados al respecto. Se pone entonces de relieve el papel que cumple la historia sobre la educación en ciencias, fundamentalmente, respecto al conocimiento sobre la biotecnología y la función que cumple la instalación de dichos conocimientos en relación con el desarrollo científico y tecnológico, políticas de educación y economía. En este sentido, se tiene una mirada anacrónica y compleja a los factores que están influyendo en la toma de decisiones sobre la inclusión de la biotecnología a la educación, al igual que en la enseñanza de la biotecnología al reconocerla como objeto de investigación en la didáctica de las ciencias.

Palabras clave: educación, biotecnología, historia, didáctica, epistemología.

\section{Abstract}

In this paper there are issues related to the advent of biotechnology in education. It is the result of search and detailed review of the first published documents about it. The role that history plays on science education is highlighted, primarily in relation to knowledge about biotechnology and the role of the installation of such knowledge regarding scientific and technological development, education and economic policies. In this sense, it has an anachronistic and complex look at the factors that are influencing the decision-making on the inclusion of biotechnology to education, as well as in biotechnology teaching to recognize it as a research object in science education.

1 Estudiante doctorado interinstitucional en educación. Universidad Pedagógica Nacional. Universidad Francisco José de Caldas. Universidad del Valle. Línea de Investigación Conocimiento Profesional de Profesor de Ciencias. robinsonroa@hotmail.com. Esta ponencia hace parte de los desarrollos alcanzados de la tesis doctoral. 
Bio-grafía Escritos sobre la Biología y su Enseñanza.

Edición Extra-Ordinaria.

Memorias del I Congreso Nacional de Investigación en Enseñanza de la Biología.

VI Encuentro Nacional de Investigación en Enseñanza de la Biología y la

Educación Ambiental. ISSN 2027 1034. P. p. 320-332.

Keywords: education, biotechnology, history, didactics, epistemology.

\section{Introducción}

En el venir y devenir del conocimiento, y con ella su enseñanza, los hechos y acontecimientos se van consolidando lentamente por lo que constituir la historia buscando dejar escrito el rastro de los intereses, estructuras, función y propiedades intrínsecas del conocimiento, condiciones y condicionamientos culturales, políticos, económicos y de educación se convierte en un elemento esencial para comprender el presente y proyectar el futuro.

En estos términos, según la teoría anacrónica, Kragh (1989), “...debería estudiarse la ciencia del pasado a la luz de los conocimientos que hoy día tenemos, y además teniendo presente esa evolución posterior, especialmente la manera en la que llegó a convertirse en lo que es en la actualidad." (p. 120). Por consiguiente, la historia, en este caso de la llegada de la biotecnología a la educación, es un asunto esencial, al igual que la epistemología y filosofía, para el diseño de contenidos y actividades de enseñanza en contextos particulares.

Teniendo en cuenta que la biotecnología se ha convertido graduablemente en un asunto de preocupación en muchos países, la educación en ciencias se ha visto como el instrumento para buscar el incremento y sostenimiento del desarrollo del conocimiento científico y de esta manera también de la economía esencialmente. Los aspectos culturales, ambientales y didácticos y con estos las características epistemológicas y filosóficas que le subyacen para la enseñanza, son en la mayoría de los casos borrados de los objetivos perseguidos por la educación en biotecnología.

La biotecnología como objeto de enseñanza no es analizada y puesta en relación con el contexto desde el cual es enseñada o se va a enseñar, por lo que se tiene la tendencia a realizar replicas a partir de las experiencias obtenidas en otros lugares. Así pues, abordar la historia sobre la inclusión de la educación de la biotecnología a las escuelas de otros países deviene fundamental para nuestro país -Colombia-, en el sentido de no ser ingenuos al responder más al qué enseñan que al cómo enseñar, por qué, para qué y desde dónde enseñar. En este sentido, se propone tratar de responder a las preguntas: ¿Cuál es la procedencia de la educación en biotecnología? y ¿Por qué enseñar biotecnología?; en concreto, no se trata de escribir la historia de la biotecnología sino más bien la historia en cuanto a su llegada a la educación, sin negar que lo 
Memorias del 1 Congreso Nacional de Investigación en Enseñanza de la Biología. VI Encuentro Nacional de Investigación en Enseñanza de la Biología y la Educación Ambiental. ISSN 2027 1034. P. P. 320-332.

anterior también se relaciona con lo que se pone como interés de análisis en el documento.

En lo que sigue entonces se presenta la manera como la biotecnología incursiona en las escuelas en Reino Unido y Estados Unidos, principalmente en el primero; y cómo en función de su experiencia, es propuesta por la Unesco para ser incluida como contenidos de enseñanza de la ciencias naturales desde la educación primaria hasta la terminación del bachillerato ${ }^{2}$. Se intenta explicitar las circunstancia sobre la cuales la biotecnología es incluida en los programas de educación en ciencias, como consecuencia de sus desarrollos científicos, impacto social y económico. Se presenta la conformación de redes internacionales, una en la Unión Europea y otra en Iberoamérica.

Con ellos, se dibuja el desarrollo que ha tenido la introducción de la biotecnología en la década del ochenta en la se percibe la preocupación del Reino Unido y Estados Unidos, por desarrollar actitudes positivas en cuanto al consumo de los productos y servicios, por cuanto esto favorecería su comercialización y la economía, dando poco énfasis a los procesos didácticos para su enseñanza y por ende la epistemología, historia y filosofía de las ciencias.

\section{Ubicando la historia sobre la enseñanza de las ciencias}

La historia de la ciencia permiten seguir el paso que constantemente la ciencia está dando desde diferentes formas de vivir y comprender el mundo, está marcada por episodios en los cuales intervienen diversos factores: poderes, creencias, mitos, tradiciones. Conocer la historia de la ciencia para la educación en ciencias constituye un elementos esencial para conectar, explicar e interpretar el pasado a la luz del presente y de las condiciones que contextos diferentes tienen para poder entender y emprender o continuar la construcción del conocimiento, sea cual sea (escolar, científico o cotidiano). "Instalar" en la enseñanza contenidos sin orientación de los objetivos que se pretenden alcanzar (aprendizaje, valores, actitudes, ideologías, etc.) es tanto como aprender por aprender, hacer por hacer sin saber para qué se aprende. Un aprendizaje y enseñanza que no construye conocimiento conociendo el origen y naturaleza de los conocimientos es tanto como construir castillos en el aire.

\footnotetext{
${ }^{2}$ En Colombia la educación de bachillerato corresponde a la educación secundaria (6ำ a 9ํ) y educación media $\left(11^{\circ}\right.$ y $\left.12^{\circ}\right)$.
} 
Memorias del 1 Congreso Nacional de Investigación en Enseñanza de la Biología. VI Encuentro Nacional de Investigación en Enseñanza de la Biología y la Educación Ambiental. ISSN 2027 1034. P. p. 320-332.

Quintanilla (2007:8) manifiesta que "...la historia de la ciencia nos permite conectar la ciencia específica con tópicos y temas propios de cada disciplina y también con otros saberes, integrando la natural interdependencia del conocimiento humano con todas las manifestaciones de la cultura en su más amplio espectro. Así mismo, resulta evidente que la ciencia 'construida y enseñada' se ha ido justificando a lo largo de la historia con argumentos epistemológicos que no son simples de analizar y nos permiten comprender escuelas de pensamiento, corrientes imperantes y maneras de entender el mundo, considerando además las experiencias de sujeto que aprenden en situaciones intencionadas de su propia cultura y valores."

Entonces, aparecen dos aspectos claves para la educación en ciencias, los cuales conforman una red a la hora de ponerlos en escena durante la acción de la enseñanza, a saber: el conocimiento sobre la procedencia (s) de los conocimientos y las características de dichos conocimientos, en términos de las formas utilizadas para su construcción, y los antecedentes que le dieron forma y contenido; igual de importante, es encontrar la manera de abordar la enseñanza del conocimiento de manera situada, teniendo como referente posturas críticas sobre la función de dichos conocimientos, que deben ser también propositivas y propender por acrecentar el entendimiento y producción del conocimiento desde el contexto en el cual es enseñado.

El llamado a hacer interpretación, análisis y propuestas a partir de los ritmos de desarrollo científico/tecnológico y social, evidentemente contrastables desde el lugar de la educación, tiene un carácter urgente. Por lo que el conocimiento se torna indispensable de interiorizar pero no de manera inconsciente, es decir, sin conocer las complejidades, su naturaleza, significado, sentido, tendencia e impacto; es decir, sin desconocer el papel que como ciudadano se tiene en la legitimación de las diferentes transformaciones de la materia prima, sean viva o muerta. Acaece pues la posibilidad, ante la realidad vivida y escrita en las publicaciones sobre estos temas, que mientras el conocimiento avanza aceleradamente la ignorancia hace igualmente lo propio. Ante esto surge la preocupación de algunos países por alfabetizar a sus presentes y, sobre todo, futuros ciudadanos y ciudadanas en los asuntos de la biotecnología.

\section{Introducción de la biotecnología a la educación}

En atención a esto y haciendo seguimiento a las primeras publicaciones derivadas de organismos internacionales se evidencia que fue la Unesco en 1990 con 
Bio-grafía Escritos sobre la Biología y su Enseñanza.

Edición Extra-Ordinaria.

Memorias del 1 Congreso Nacional de Investigación en Enseñanza de la Biología.

VI Encuentro Nacional de Investigación en Enseñanza de la Biología y la

Educación Ambiental. ISSN 2027 1034. P. p. 320-332.

participación de la Comisión de Educación en Biología de la International Union of Biological Science (IUBS) ${ }^{3}$, quien de manera oficial publicó el libro Teaching Biotechnology in School - editado por Joseph D. Mclnerney-, que fue en gran parte resultado de una conferencia en mesa redonda sobre biotecnología y educación, llevada a cabo en 1989 en Asendorf, República Federal Alemana, en la cual participaron universidades de varios países como Bulgaria, Singapur, India, Alemania, Finlandia, China y Estado Unidos de América. Antes de que la Unesco publicara el libro, Wymer (1992) subraya que la biotecnología había ganado una significativa presencia en las escuelas y universidades del Reino Unido durante la década de los ochenta como resultado de la iniciativa del gobierno central, instituciones y ayudas individuales, aportando de esta manera a la alfabetización biotecnológica. Años antes, Wymer (1986) escribe que David Micklos, del Cold Spring Harbort Laboratory, en New York (Reconocido centro internacional de investigación en genética), había señalado la escases de enseñanza de la biotecnología en las escuelas, por lo que planeó para su difusión realizar viajes desde las escuelas de California hasta las de New Hampshire en una camioneta plateada la cual Ilamó "the Vector Mobile DNA Laboratory", con el propósito de que profesores y estudiantes pudieran recibir capacitación y tuvieran la posibilidad de visitar el laboratorio y realizar prácticas sobre técnicas de recombinación de ADN al igual que observar las instalaciones y exhibiciones de informática.

Cuenta Wymer (1992) que éste antecedente sirvió como parte de la justificación en el Reino Unido para la creación del National Centre for Biotechnology Education $^{4}$ (NCBE) del cual él fue cofundador y director en 1985, al igual que editor de la revista, Biotechnology Education ${ }^{5}$, la otra parte de la justificación de la creación del centro fue un informe sobre una investigación orientada por el Department of Trade and Industry (DTI) del Reino Unido durante un año (19841985) para conocer el nivel de conciencia de la biotecnología en las escuelas. Una parte de las conclusiones del informe es que "Si bien muchos profesores conocen

\footnotetext{
${ }^{3}$ Comisión para la Educación Biológica de la Unión Internacional de Ciencias Biológicas, se creó en los años setenta como brazo educativo de la Unión. Su papel principal es formular, iniciar y facilitar métodos eficaces para mejorar la educación en las ciencias biológicas y áreas afines, incluidas las aplicaciones e implicaciones de los estudios biológicos. La comisión tiene el compromiso de contribuir a la difusión internacional y el intercambio de información sobre la educación biológica, lleva a cabo conferencias internacionales cada cuatro años.

http://translate.google.com.co/translate?hl=es\&langpair=en\%7Ces\&u=http://www.iubs.org/prg/bioed.html

${ }_{5}^{4}$ Primero fue llamado National Centre for School Biotechnology.

${ }^{5}$ Esta es una revista internacional trimestral, lanzada por Pergamon Press en 1989, la función principal es proporcionar un foro para profesores de los cursos de la biotecnología para el intercambio de ideas, conceptualizar y probar los protocolos de laboratorio. La revista también lleva a revisar artículos de opinión (Wymer, 1992).
} 
Memorias del I Congreso Nacional de Investigación en Enseñanza de la Biología. VI Encuentro Nacional de Investigación en Enseñanza de la Biología y la Educación Ambiental. ISSN 2027 1034. P. P. 320-332.

del potencial e importancia económica de la biotecnología, y están interesados en la incorporación de aspectos de la misma en su enseñanza, pocos tienen la formación, experiencia o incentivos educativos para formar en los estudiantes una conciencia sobre el tema, e incorporar en los planes de estudio las técnicas y los procesos, la subyacente ciencia y el contexto económico de la biotecnología". Adicionalmente se encontró evidencia de que la inclusión de palabras como artificial, clonación, terapia génica, ingeniería genética y productos industriales, en cualquiera de las declaraciones dadas generaba desaprobación.

El NCBE y otras entidades tales como The University of Reading School of Education; The London Centre for Biotechnology; y la Division of Education at the University of Sheffiel, apoyadas por industrias locales, sociedades científicas o instituciones académicas, se interesaron por que la enseñanza de la biotecnología mejorara en las escuelas, particularmente en la secundaria y media, por lo que se dedicaron a elaborar curso de capacitación a profesores, poner en discusión asuntos polémicos, paquetes de experimentos y material de divulgación de aspectos biotecnológicos.

James Watson, en un boletín de noticias de la edición inaugural de la NCSB, elabora un escrito titulado School Biotechnology: An issue of Primary importance, en el cual señala que el "National Centre for School Biotechnology representa el primer compromiso nacional para actualizar la enseñanza pre-universitario teniendo en cuenta el dramático aumento de la ciencia del ADN. ${ }^{6}$

Así pues, continuando con lo descrito por Wymer (1992) este centro ha tomado el liderazgo en Europa y en el Lejano Oriente en la promoción de la enseñanza de la biotecnología en las escuelas. De hecho fue invitado por la Unesco para participar en la conferencia de mesa redonda realizada en 1989 en Asendorf, Alemana. Así también fue promotor de la European Initiative on Biotechnology Education (E.I.B.E), la cual es apoyada con fondo de la Comision de las Comunidades Europeas, unos reglones más adelante se tratará de describir un poco más esta iniciativa.

Dean Madden del NCBE, quien hace parte del grupo que reemplazó a Paul Wymer en 1990, en el 2005 en su publicación The English patient Biotechnology education in the UK (El paciente inglés, educación en Biotecnología en el Reino

\footnotetext{
${ }^{6}$ En: http://www.ncbe.reading.ac.uk/NCBE/NCBE/history.html. Según comenta Dean Madden, Watson, estuvo interesado en el intento de establecer un "centro de aprendizaje de ADN", similar al NCSB, en su laboratorio de Cold Spring Harbor.
} 
Bio-grafía Escritos sobre la Biología y su Enseñanza.

Edición Extra-Ordinaria.

Memorias del 1 Congreso Nacional de Investigación en Enseñanza de la Biología.

VI Encuentro Nacional de Investigación en Enseñanza de la Biología y la

Educación Ambiental. ISSN 2027 1034. P. p. 320-332.

Unido), pone en discusión de manera critica los verdadero alcances del programa y se pregunta ¿Qué lecciones se pueden aprender de la experiencia inglesa que pueda servir de modelo para otras iniciativas?

Haciendo referencia a lo anterior destaca la disminución en la cobertura de los currículos escolares en biotecnología desde mediados de los 80 , cuando fue el Reino Unido (aunque sea muy brevemente) un líder mundial reconocido, hasta el presente. De manera parecida a como Wymer (1992) ya lo había presentado, Madden presenta brevemente los materiales de lectura y de laboratorio, videos y programas informáticos desarrollados, laboratorios móviles, entre otras cosas, aspecto que se resaltó con anterioridad en este escrito. Además, describe que varios profesores fueron adscritos a un proyecto en la Universidad de Sheffield por tres años para que se estudiaran las metodologías de enseñanza, prestando especial atención a cómo la experiencia de los profesores afectaba su práctica en el aula al desarrollar temas polémicos; finalmente declara que existía falta de consenso sobre la definición de la biotecnología llevada a la educación, lo cual generaba con el tiempo poco interés y temor, de manera generalizada, por los aditivos en los alimentarios.

El proyecto en Sheffield estaba más interesado en la investigación en educación para capacitar en aspectos de la biotecnología, en lugar de la educación biotecnológica por sí misma. Así mismo, el autor informa sobre varios cursos y estrategias de difusión de la biotecnología que con el tiempo no continuaron por falta de financiación ${ }^{7}$, al igual que hubo poco acuerdo sobre los contenidos a desarrollar en la secundaria en cuanto a si sería enseñada la biotecnología, en sentido estricto o solamente conceptos básicos. En cuanto esto, y buscando llegar a un conceso, se realiza una conferencia nacional en la Universidad de Kent en Canterbury en 1986 a la cual se invitó a docentes, asesores de ciencia de autoridad en educación local, representantes de la industria y las universidades. Previo a la conferencia ya se habían celebrado reuniones similares en Cardiff y Londres. Comenta Madden, que el profesor Paul Black, del King's College London, advirtió sobre la necesidad de definir los planes de estudio sobre la biotecnología en la escuela y cómo esta podría ser evaluada.

En definitiva, Madden señala que en 1989, el gobierno británico impuso el plan de estudios nacional en Inglaterra y Gales, especificando lo que se iba a enseñar a

\footnotetext{
${ }^{7}$ En 1990 Paul Wymer es retirado del centro tomando la dirección John Grainger, teniendo como asistentes a John Schollar and Dean Madden. En 1992 todos los fondos del gobierno habían cesado y el NCBE se dejó a su suerte. En: http://www.ncbe.reading.ac.uk/NCBE/NCBE/history.html
} 
Memorias del 1 Congreso Nacional de Investigación en Enseñanza de la Biología. VI Encuentro Nacional de Investigación en Enseñanza de la Biología y la Educación Ambiental. ISSN 2027 1034. P. p. 320-332.

todos los niños entre 5 y 16 años de edad. Esta versión del plan de estudios nacional había incorporado solo algunos aspectos de la biotecnología. En términos generales, las políticas en educación fueron variando y cambiando la dinámica de las escuelas lo cual afectó las primeras iniciativas de introducción de la biotecnología en la escuela. En orden a esto se combinaron aspectos atinentes al temor de los empresarios sobre el mercado, la percepción pública y la generación de tensión con las escuelas.

Otro aspecto importante en la consolidación de la biotecnología en la escuela fue la creación de la European Initiative for Biotechnology Education (EIBE) en 1991, con el objetivo de desarrollar habilidades, aumentar la comprensión y facilitar el debate público informado a través de una mejor enseñanza de la biotecnología en escuelas, institutos de enseñanza secundaria y universidades de la Unión Europea. Su principal actividad ha sido generar materiales didácticos para personas entre los 16 -19 años de edad. Las unidades de enseñanza elaboradas consisten en un conjunto de actividades con variedad de protocolos experimentales, actividades prácticas, juegos de rol, información y debates. A estos materiales se tiene acceso en internet en varios idiomas, se pueden encontrar 20 unidades elaboradas desde 1996 hasta el 2000.

La EIBE se había convertido en una activa red europea, multidisciplinar, conformada por algunos expertos en educación, procedentes de 20 centros ubicados en varios países europeos, los cuales son: Bulgaria, Estonia, Polonia, Suiza, República Checa, Grecia, Bélgica, Dinamarca, Alemania, Irlanda, España, Francia, Italia, Luxemburgo, Países Bajos, Suecia, Reino Unido.

Para el caso de Latino América y España se ha configurado, desde el 2006, la Red Iberoamericana de Educación en Biotecnología Agroalimentaria, BIOEDUCAR ${ }^{9}$, con el apoyo de CYTED y RedBio/FAO ${ }^{10}$, esta tiene como principal objetivo coordinar fortalezas y oportunidades disponibles de educación en biotecnología agroalimentaria en Iberoamérica, para fomentar y facilitar la comunicación entre científicos y sociedad en los campos de las ciencias de la vida. También se pretende el desarrollo de proyectos conjuntos en diferentes instancias de trabajo, discusión, exposición y análisis, en beneficio de la calidad y

\footnotetext{
${ }^{8}$ En: http://www.eibe.info/

${ }^{9}$ En: http://www.upv.es/VALORES/Documentaci\%F3n/CYTED/Convocatoria.pdf

${ }^{10}$ El apoyo de CYTED y RedBio/FAO está acordado del 2004 al 2007, a partir de esta fecha se espera poder contar con otras entidades para la sostenibilidad de la Red.
} 
Bio-grafía Escritos sobre la Biología y su Enseñanza.

Edición Extra-Ordinaria.

Memorias del 1 Congreso Nacional de Investigación en Enseñanza de la Biología.

VI Encuentro Nacional de Investigación en Enseñanza de la Biología y la

Educación Ambiental. ISSN 2027 1034. P. p. 320-332.

pertinencia de la enseñanza, al igual que un cambio de percepción pública de las nuevas tecnologías aplicadas a la producción de alimentos.

En este marco, la red espera generar intercambios de experiencias, definir y coordinar temas de investigación de relevancia social y/o económica; desarrollar programas neutros, con base científica y bioética para la educación en biotecnología agroalimentaria (escuela primaria, secundaria, profesionales de la salud y la educación, comunicadores sociales, gestores de política y público en general); proponer el desarrollo y presentación de un proyecto conjunto. En esta red participan 9 países de Iberoamérica: Argentina, Brasil, Bolivia, Chile, Cuba, Ecuador, España, México y Venezuela.

Hasta este punto se puede decir que la biotecnología es un tema que empieza a ser puesto en análisis de manera conjunta con la educación en el siglo XX desde inicio de los ochenta en el Reino Unido ${ }^{11}$ y Estados Unidos, teniendo estos dos países acercamiento para compartir, en este momento, las experiencias desarrolladas.

Particularmente la NCBE en el Reino Unido, toma un papel importante en el liderazgo de los análisis realizados en la conferencia que tuvo lugar en Alemania en 1989; bajo este evento el estadounidense Joseph D. McInerney, director de la Biological Sciences Curriculum Study (BSCS) ${ }^{12}$, participa activamente en 1990 en la edición del primer libro Teaching biotechnology in schools, auspiciado por la Unesco.

No sobra expresar, que la primera publicación se encuentra referenciada por Wymer (1990), a través de un informe de trabajo de la Royal Society en 1981 titulado "Biotechnology and education", el cual se encuentra en forma de libro y de artículo en Journal of Biological Education, 1982.

Por su parte, Lui y Chan (1999) hacen otra cita de la Royal Society (1981) bajo el título de "Biotechnology - an interdisciplinary curriculum for schools", publicado en School Science Review.

\footnotetext{
${ }^{11}$ El primer documento que hasta este momento se ha encontrado corresponde a un informe de trabajo de la Royal Society en 1981 titulado Biotechnology and education.

${ }^{12}$ Barker (1990) aclara que la BSCS ha tenido una importante influencia en USA y en muchos otros países por más de 30 años. Fue creada en 1958 con la meta de rejuvenecer y reestructurar la bilogía del grado décimo de las escuelas americanas. Dentro de los productos alcanzados están tres libros de biología para la educación media, comercializados en 1963, identificados por el color de sus cubiertas: la amarilla, que enfatiza en los organismos en términos de su estructura y función; la azul, que se enfoca en la bilogía molecular; y la verde que desarrolla un curso de biología con visión ecológica.
} 
Memorias del I Congreso Nacional de Investigación en Enseñanza de la Biología.

VI Encuentro Nacional de Investigación en Enseñanza de la Biología y la

Educación Ambiental. ISSN 2027 1034. P. p. 320-332.

\section{Unas primeras conclusiones}

Al respecto se puede decir que lo que fundamentalmente los países han buscado al introducir la biotecnología en la escuela, es evitar que las personas entraran en desconfianza con la aplicación de los conocimientos científicos para la producción de alimentos, medicamentos, etc. Al referente, Taguenca (2008), expresa que las nuevas biotecnologías representan una gran esperanza para el ser humano. Sin embargo, la gente tiene una percepción respecto a ellas ambivalente, donde el bien y el mal que suponen están juntos.

En concordancia, Roa y Valbuena (2009) haciendo un balance sobre la importancia de la educación en biotecnología, explicitan la necesidad de realizar más investigaciones tanto en lo atinente a la enseñanza y el aprendizaje de conceptos específicos biotecnológicos, como en lo relacionado con referentes transversales, lo cual conlleva a la realización de estudios sobre su historia y epistemología, así como también de naturaleza filosófica.

Es necesario reconocer las historia recorrida por otros países sobre la llegada de la biotecnología a la educación para identificar las circunstancias sobre las cuales se tomaron las decisiones y los resultados alcanzados, y a partir de estos proponer objetivos que respondan las necesidades y circunstancias del país o región, esto es cultura, biodiversidad, posición geográfica, alimentaria, empleo, necesidad de profesionales tanto para la enseñanza y su respectiva investigación, como para la investigación en biotecnología.

Se puede entender que las actividades realizadas por el Reino Unido y los Estados Unidos de América se fueron replicando en otros países a través de la conformación de redes y/o centros especializados, los pasos clave para esto fue el evento realizado en Alemania en 1989, y el libro Teaching biotecnology in schools.

Por las características culturales e ideológicas de los países es una condición imprescindible analizar las decisiones que se vayan a tomar respecto a la introducción de contenidos de enseñanza, nos e pueden replicar las experiencias obtenidas por otros países que tiene otras tradiciones y creencias, acerca de lo material, los ciudadanos, lo vivo y la vida, entre otros aspectos.

Al desarrollar la didáctica de la biotecnología como un objeto de su investigación y de su enseñanza es fundamental identificar las condiciones que dieron cuenta de este conocimiento, así por ejemplo, no es lo mismo problematizar la enseñanza de 
Memorias del 1 Congreso Nacional de Investigación en Enseñanza de la Biología. VI Encuentro Nacional de Investigación en Enseñanza de la Biologia y la Educación Ambiental. ISSN 2027 1034. P. P. 320-332.

la biotecnología tradicional a la biotecnología moderna; enseñarla como una multidisciplina o como una interdisciplina o transdisciplina, o incluso como una ciencia, enseñar sus contenido desde la tecnología que desde la biología, la química o la física.

Vale poner de relieve en este punto, que es necesario para Colombia incluir la didáctica de la biotecnología en todos los niveles de educación, pero no como en el apartado destinado a la educación en el libro publicado por Conciencias: La biotecnología, motor de desarrollo para la Colombia de 2015, el cual intenta copiar fielmente los resultados y estrategias utilizadas en otros países para instalar la biotecnología en las escuelas y en la sociedad, especialmente a través de folletos, boletines, medios de comunicación, y capacitación a periodistas, desconociendo la naturaleza de la didáctica de la biotecnología -incluso la de la misma biotecnología-. Sin duda esto tiene mucho que ver con el tipo de ciudadano que se quiere formar: consumista, ilustrado, investigador, etc.

Es evidente que el interés de traer la biotecnología a la escuela surge en la escuela sino más bien del exterior a ella, fundamentalmente del mercado.

\section{Agradecimientos}

Se agradece al Doctor Édgar Orlay Valbuena Ussa, coordinador de la línea de investigación Conocimiento Profesional del Profesores Ciencias, por los aportes realizados en la elaboración del taller.

\section{BIBLIOGRAFÍA}

- BARKER, J. (1990). Biological Sciences Curriculum Study. Journal of Biological Education. 24, (3), 142-143.

- European Initiative for Biotechnology Education (EIBE) (2000). Julio, noticias 8. En: http://www.ipn.uni-kiel.de/eibe/NEWS8ES.PDF

- instituto colombiano paRa el desaRRollo de la CIENCIA Y LA TECNOLOGÍA "FRANCISCO JOSÉ DE CALDAS" COLCIENCIAS. UNIVERSIDAD NACIONAL DE COLOMBIA, y CORPOGEN, INVESTIGACIÓN Y BIOTECNOLOGÍA EN COLOMBIA. (2008). La 
Memorias del I Congreso Nacional de Investigación en Enseñanza de la Biología. VI Encuentro Nacional de Investigación en Enseñanza de la Biología y la Educación Ambiental. ISSN 2027 1034. P. p. 320-332.

biotecnología, motor de desarrollo para la Colombia de 2015. Bogotá, Colombia.

- KRAGH, H. (1989). Introducción a la historia de la ciencia. Barcelona: Editorial Crítica.

- LUI, K y CHAN, S. (1999). Biotechnology education for teacher trainees. New Horizons in Education. The Journal of Education Hong Kong Teachers' Association (HKTA). 40, $109-116$.

- MADDEN, D. (2005). The English patient Biotechnology education in the UK.

En: CSERMELY, P; KORCSMÁROS, T; y LEDERMAN, L. Science Education: Best Practices of Research Training for Students under 21. NATO Science Series, V: Science and Technology Policy. 47, 143-152.

- MCLNERNEY, J. (editor). (1990). Teaching Biotechnology in School. Paris: UNESCO.

- QUINTANILLA, M. (2007). La historia de la ciencia como una estrategia para aprender a pensar sobre el mundo. En: QUINTANILLA, M. Historia de la ciencia. Aportes para la formación del profesorado. Volumen I. Santiago de Chile. Editorial Conocimiento.

- ROA, R Y VALBUENA, E. (2009). Hacia la estructuración de la educación en Biotecnología como problema didáctico de investigación. Memorias: Primer Congreso Nacional de Investigación en Educación en Ciencias y Tecnología. Asociación Colombiana para la Investigación en Ciencia y Tecnología, Bogotá.

- RED IBEROAMERICANA DE EDUCACION EN BIOTECNOLOGIA AGROALIMENTARIA. (2005). BIOEDUCAR. En: http://www.upv.es/VALORES/Documentaci\%F3n/CYTED/Convocatoria.pdf

- ROYAL SOCIETY (1981). Biotechnology - an interdisciplinary curriculum for schools. School Science Review. 68, 699-701.

- ROYAL SOCIETY. (1981). Biotechnology and education. Informe grupo de trabajo. 
Memorias del I Congreso Nacional de Investigación en Enseñanza de la Biología. VI Encuentro Nacional de Investigación en Enseñanza de la Biología y la Educación Ambiental. ISSN 2027 1034. P. P. 320-332.

- ROYAL SOCIETY. (1982). Biotechnology and education. Journal of Biological Education, 16 (1) 10-12. En: http://www.eric.ed.gov/ERICWebPortal/search/detailmini.jsp?_nfpb=true\&_\& ERICExtSearch_SearchValue_0=EJ266247\&ERICExtSearch_SearchType_ $0=$ no\&accno $=E J 266247$

- TAGUENCA, J. (2008). La opinión de los españoles ante las nuevas biotecnologías. Papers. 88, 31-44.

- WYMER, P. (1986). A driving force in biotechnology. New Scientist 1525, 63.

- WYMER, P. (1992). Biotechnology in schools: the UK experience. World Journal of Microbiology and Biotechnology. 8, 473-476.

- WYMER, P. (1990). Considerations for implementation. En: Mclnerney, J. (editor). Teaching Biotechnology in School. Paris. Unesco. 